The radiated power from the TCA Tokamak over a wide range of ohmically heated conditions

This article has been downloaded from IOPscience. Please scroll down to see the full text article.

1987 Plasma Phys. Control. Fusion 2927

(http://iopscience.iop.org/0741-3335/29/1/002)

View the table of contents for this issue, or go to the journal homepage for more

Download details:

IP Address: 128.178.125.142

The article was downloaded on 18/02/2013 at 08:50

Please note that terms and conditions apply. 


\title{
THE RADIATED POWER FROM THE TCA TOKAMAK OVER A WIDE RANGE OF OHMICALLY HEATED CONDITIONS
}

\author{
B. Joye, J. B. Lister, J.-M. MORET and S. NOWAK* \\ Association Euratom-Confédération Suisse, Centre de Recherches en Physique des Plasmas, \\ Ecole Polytechnique Fédérale de Lausanne, 21, av. des Bains-1007 Lausanne/Switzerland \\ * Department of Physics, University of Fribourg, Pérolles, Fribourg/Switzerland \\ Received 7March 1986; and in revised form 27 June 1986)
}

\begin{abstract}
We present detailed measurements of the radiated power loss from the TCA Tokamak using a 16-channel radiation bolometer array. The plasma density has been varied over a wide range up to the high density disruption limit, at several magnetic fields and plasma currents. The fractional radiated power is a well correlated function of the average electron drift speed, and is independent of the toroidal magnetic field. The occurrence of disruptions does not appear to be simply correlated with the total fractional radiated power, but more with the radiated power fraction on axis. The injection of light impurities clearly illustrates that there is no simple universal "law" which relates the disruption limit in TCA to the measurable radiated power loss. No evidence of any disruption "precursor" has been seen in the bolometer data.
\end{abstract}

\section{INTRODUCTION}

THE EXISTENCE of a high density limit to the operational range of a Tokamak was established by MUraKami et al. (1976) in terms of the reduced parameter $\bar{n}_{e} R / B_{\varphi}$, where $\bar{n}_{e}$ is the line-averaged electron density, $R$ is the major radius of the discharge and $B_{\varphi}$ is the toroidal field. The importance of this limit on future Tokamak operation was stressed at the same time by GiBson (1976). Since these early observations, this limit has persisted for ohmically heated discharges, and remains one of the best established "facts" of Tokamak operation. The value of $\bar{n}_{e} R / B_{\varphi}$ obtained on different ohmically heated tokamaks ranges from $4-6 \times 10^{19}$ Weber $^{-1}$.

A large effort has been devoted to understanding or predicting this operational limit, mainly in terms of the effect of impurity radiation on the thermal balance, already proposed at the time by both Murakami and Gibson. The reduced parameter $\mathrm{B}_{\varphi} / R$ is proportional to the current density on axis, $j(0)$, for a fixed safety factor on axis $\left(\mathrm{q}_{0}\right)$, via $j(0)=B_{\varphi} / R \times 2 / \mu_{0} q_{0}$, and thereby related to the ohmic heating power density on axis, $P_{\text {oh }}(0)=E(0) j(0)$.

Figure 1 shows the part of the operational diagram, $1 / q_{a}$ versus $\bar{n}_{e} R / B_{\varphi}$, for the safety factor at the limiter radius, $q_{a}$, greater than three. These steady state data were obtained using $\mathrm{SiC}$ coated limiters in TCA, whose parameters are: $R, a=0.61,0.18$ $\mathrm{m}, B_{\varphi}=0.78-1.51 \mathrm{~T}, I_{p} \leqslant 170 \mathrm{kA}$ and $n_{e}(0) \leqslant 1.5 \times 10^{20} \mathrm{~m}^{-3}$. This parameter range is fairly typical of medium-sized circular cross-section tokamaks. For $q_{a}>3$, the density limit is a function of $q_{a}, q_{a} \bar{n}_{e} R / B_{\varphi}$ being constant. For a given shape of the radial density profiles this relationship is equivalent to $e N_{e} / I_{p}=$ constant, where $N_{e}=\int n_{e}(r) \mathrm{d} S$, and is therefore a measure of the inverse of the average electron drift speed. The universal form of this boundary presents a challenge to predictive models.

Several authors have explained this limit. REBUT and GREEN (1977) introduced a radial transport model, and derived a density limit. ROBERTs (1983) derived a limit 


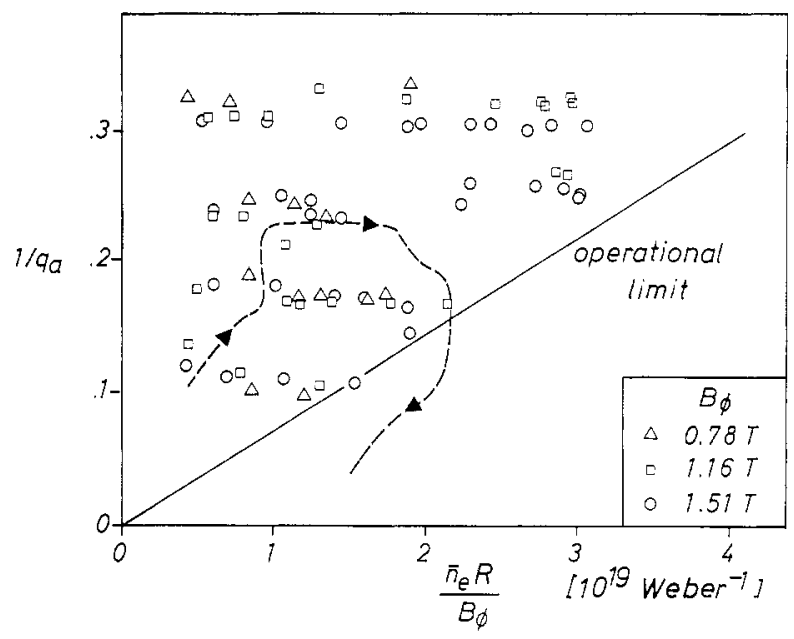

FIG. 1.-The operation diagram for stationary conditions in TCA for $q_{a}>3$ and for three different magnetic fields. The dashed line is a temporal evolution.

from the effect of edge-cooling. Perkins and Hulse (1985) treated the problem choosing an a priori limit of $\int P_{\mathrm{rad}} \mathrm{d} V / \int P_{\mathrm{o} h} \mathrm{~d} V=80 \%$, integrated within the $q(r)=2$ surface. Neither Roberts nor Perkins derived the explicit $1 / q_{a}$ dependence from the radiative balance alone, since the behaviour inside $q(r)=2$ is not simply determined by $q_{a}$. WESSON et al. (1985) have derived a limit for the JET Tokamak in which the $1 / q_{a}$ dependence is explicit, as well as the $B_{\varphi} / R$ dependence. The limit is given by the onset of thermal insulation from the limiters, i.e. $\int P_{\mathrm{rad}} \mathrm{d} V / \int P_{\mathrm{o} h} \mathrm{~d} V=100 \%$, although detached discharges can be stable for a long time. This model assumes that the impurity concentration is independent of density, and that the radiation is limited to a narrow band near the plasma edge, clearly an approximation which is only valid for a discharge in which the impurities are fully stripped at the centre, whereas the density limit is found to exist in all conditions. The assumption that the density profile is density-independent is not valid for TCA. The fact that the radiation profiles can be so different across the range of machines with the same form of the density limit is therefore extremely important.

In their paper, PERKINS and Hulse (1985) remarked on the lack of experimental data, especially the radiated power profiles to compare with a model. In this paper we present one such set of experimental data, indicating that certain gross assumptions inherent in some models are not universally appropriate.

The plan of the paper is as follows. In Section 2 we describe the experimental conditions in which the data were taken. In Section 3 these data are analysed, searching for some underlying structure. In Section 4 we present the temporal evolution of the profiles of radiated power, up to the disruption itself.

\section{EXPERIMENTAL CONDITIONS}

The data described in this paper were obtained with either $\mathrm{H}_{2}$ or $\mathrm{D}_{2}$ as the filling gas. The discharges were all full-aperture $(0.18 \mathrm{~m})$, limited by four separate $\mathrm{SiC}$ coated graphite limiters in a "picture frame" configuration. We varied the controllable operational parameters, $B_{\varphi}, \bar{n}_{e}, I_{p}$ as widely as possible. In addition we ran 
discharges with various low- $Z$ gases puffed in during the plasma current flat-top, for comparison with the "pure" filling discharges. In these cases the impurity injection increased the electron density by a factor $2-3$.

The diagnostics used were conventional. The central chord-averaged density was measured by a $140 \mathrm{GHz}$ interferometer, the radial density profile by an 8-channel $447 \mu \mathrm{m}$ interferometer and the electron temperature, by a single point single pulse Thomson scattering system. The most important diagnostic was a 16-channel radiation bolometer which uses semiconducting elements as temperature-dependent resistances (JoYE et al., 1985). The time resolution of the detectors is of the order of one millisecond. This instrument views a set of equally spaced chords across the plasma through a narrow horizontal slit, Fig. 2. The whole of the lower half of the

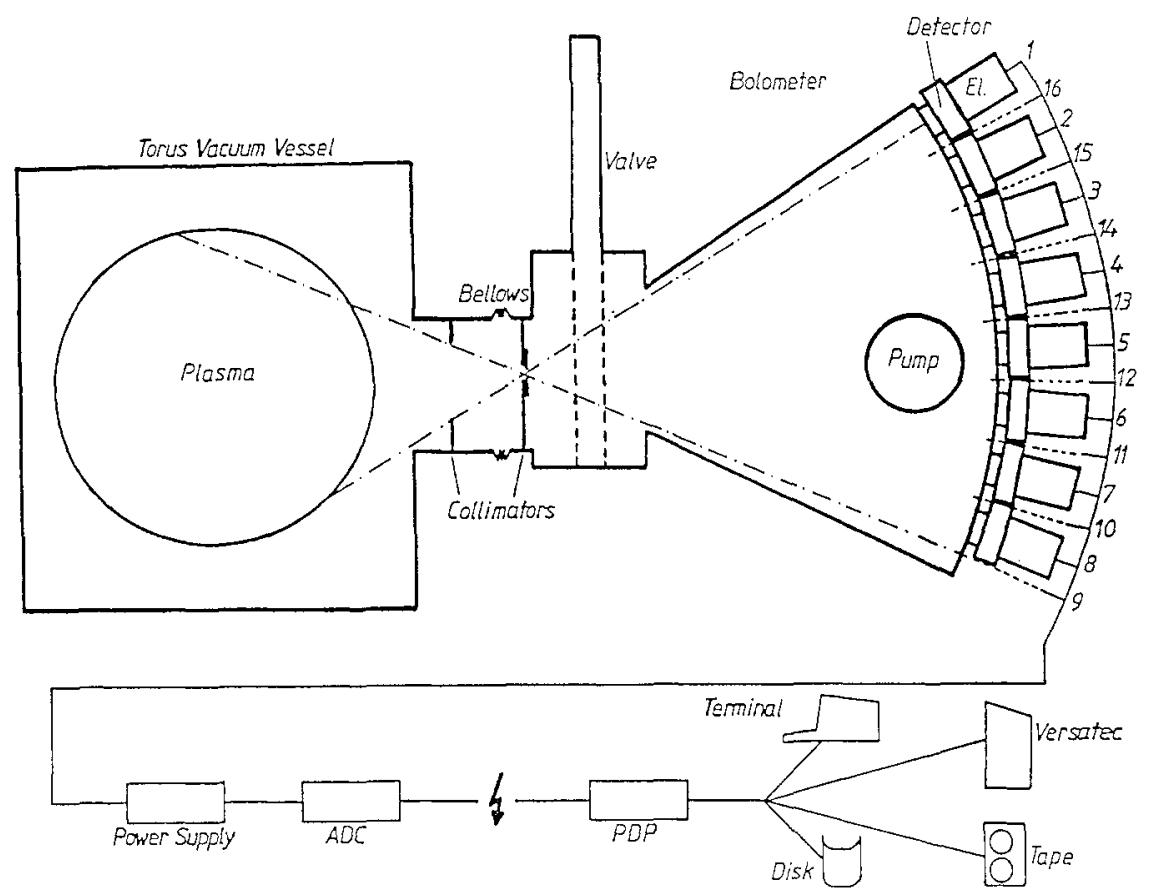

FIG. 2.-A schematic view of the multi-channel bolometer diagnostic.

plasma, and up to $0.15 \mathrm{~m}$ of the upper half is accessible. The major problem of such a system is its inability to detect any in-out poloidal asymmetry. Specifically the occurrence of a "Marfe", as observed at high density on some machines (NIEDERMEYER et al., 1983; LIPSCHULTZ et al., 1984), would render the interpretation difficult. We have examined the FIR interferometer data for evidence of "Marfe" activity on TCA. The viewing chords of this instrument are vertical, with an inner channel tangent radius of $0.155 \mathrm{~m}$ which would detect a "Marfe" if it occurred. We have not seen such an event. Marfe activity is generally accompanied by a large local increase in $\mathrm{H}_{\alpha}+\mathrm{D}_{\alpha}$ emission, which we have not seen on a horizontally-viewing monitor. Also none of the lines from low- $Z$ ionisation states, which would become more intense during a Marfe, have shown any anomalously high activity. We therefore proceed on the basis of poloidal symmetry. 
In Section 3 we will restrict ourselves to a study of quasi-stationary conditions. The condition of constant plasma current is particularly important, since it is observed that the density limit achieved in a stationary state can be exceeded for $\mathrm{d} I_{p} / \mathrm{d} t<0$, for example when the loop voltage is cut at the end of the discharge. The dotted line in Fig. 1 shows one example of a trajectory in the operation diagram, which clearly crosses the stationary state limit. We ignore those disruptions preceded for more than one confinement time by a large increase in tearing mode activity. These disruptions belong to the sets of $q$-limits of the operation diagram.

\section{ANALYSIS OF THE BOLOMETRIC DATA}

Figure 3 shows the variation of the volume integrated radiated power normalized to the ohmic power as a function of the inverse average drift speed for all the plasma currents and toroidal fields shown in Fig. 1 . The measurements were taken at $\sim 100$ $\mathrm{ms}$ into the discharge, well within the current flat-top. The filling gas was deuterium.

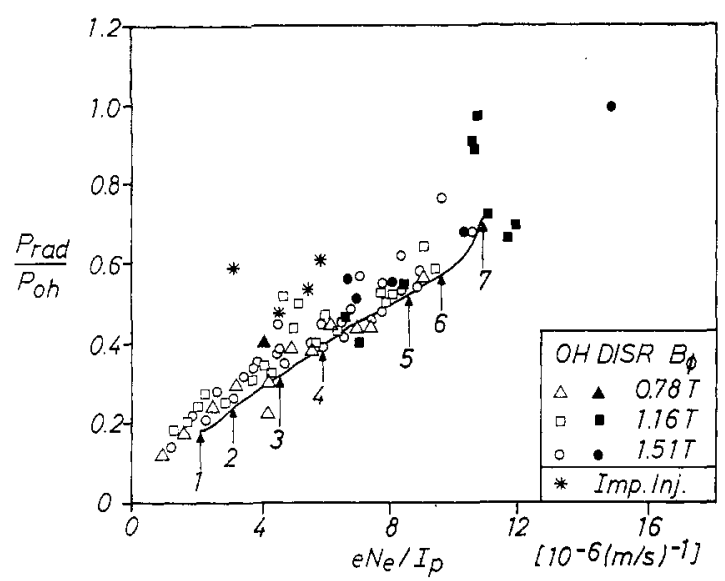

FIG. 3.--The normalised radiated power loss as a function of the inverse average drift speed. The solid line illustrates the evolution of this function during one discharge.

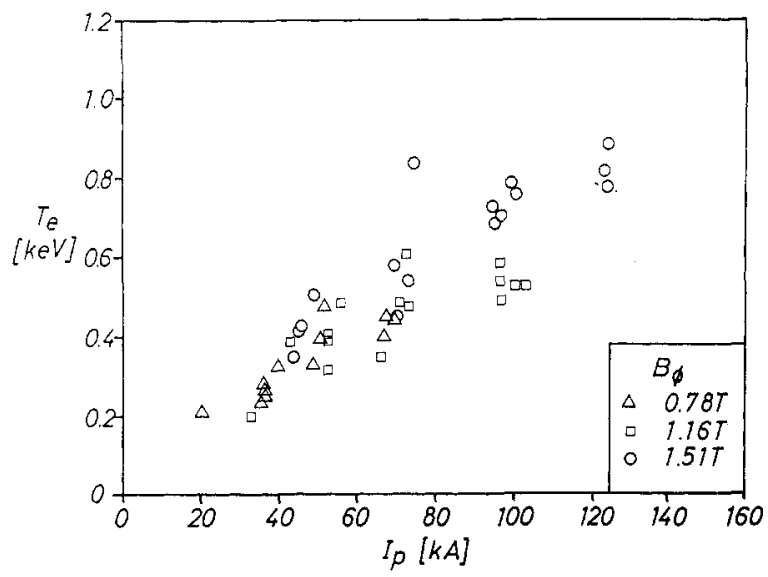

FIG. 4.-The dependence of the electron temperature on the plasma current for several values of the toroidal field. 
Each symbol represents a different magnetic field. It is important to note that the integral of the observed radiated power is not the total radiated power from the plasma, since we are only observing the plasma at one toroidal location $\sim 130^{\circ}$ away from the limiter sector. The multichannel bolometer measures the toroidally symmetric part of the radiated power excluding the radiated power local to the limiter, which is greater, mainly due to impurities which are recycled or introduced at the limiter before they have reached an ionization level at which they can complete a toroidal transit. The value of the magnetic field has no effect on the distribution, which is possibly related to the lack of dependence of $T_{e}(0) / I_{p}$ on the magnetic field, illustrated in Fig. 4. The independence of the fractional radiated power loss on $B_{\varphi}$ therefore implies an independence on $q_{a}$. Figure 3 shows discharges with impurity puffing which depart from the trend for the "pure" $\mathrm{D}_{2}$ discharges. Discharges terminating benignly and those which terminated in a density disruption are indicated by open and filled symbols respectively. The solid line illustrates the trajectory of one particular shot slowly evolving in density. Although the disruptions occur generally at high values of $e N_{e} / I_{p}$, i.e. at the high density limit, we have not isolated a fixed disruption "threshold". The disruptions occur between values of $\int P_{\mathrm{rad}} \mathrm{d} S / \int P_{\mathrm{oh}} \mathrm{d} S=$ $40-100 \%$, frequently below the threshold seen by Wesson et al. (1985), although we only detect the toroidally symmetric part of the radiated power loss, as discussed.

With the poloidal symmetry caveat, we have inverted the radiated power profile, to estimate $P_{\text {rad }}(0)$. We have checked the sensitivity to the assumptions on the emission at the edge, by varying the radius at which the emitted power is assumed to be zero. This results in a negligible uncertainty in the estimate of total radiated power loss and only a small uncertainty in the radiated power loss on axis. The problem of toroidal symmetry is no longer important since, near the axis, the limiter position has no influence on the toroidal variation of the radiated power. Figure 5 shows both $P_{\text {rad }}$ and $P_{\text {oh }}$ as local and radially integrated values, for a discharge with

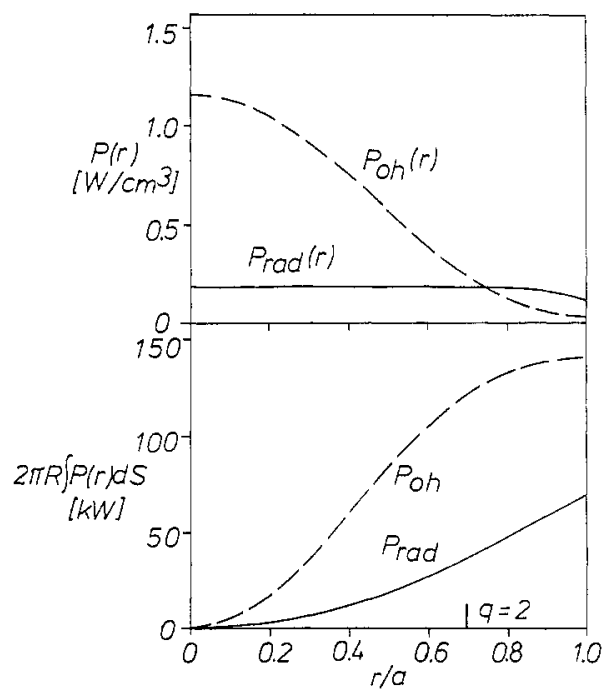

FIG. 5.-The estimated radial values (local and integrated) of ohmic and radiated power, $\left(\mathrm{D}_{2}, B_{\varphi}=1.16 \mathrm{~T}, I_{p}=83 \mathrm{kA}, \bar{n}_{e}=10^{19} \mathrm{~m}^{-3}\right.$.) 


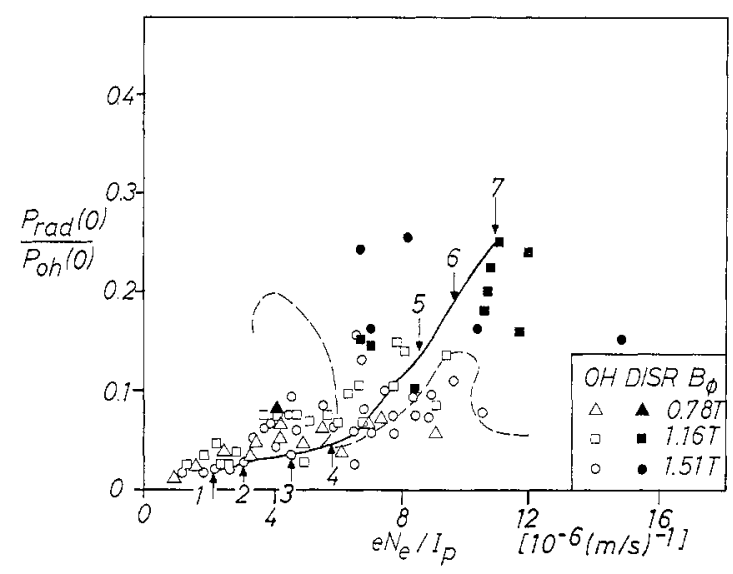

FIG. 6.-The normalised axial radiated power loss as a function of the inverse average drift speed. Several discharge evolution curves are shown as solid or dotted lines.

a density of $\sim 80 \%$ of the disruption limit. $P_{\text {oh }}(r)$ is a crude estimate based on assumed profiles of $E(r)$ and $j(r)$, and unfortunately cannot be measured directly. Figure 6 shows the dependence of the reduced axial radiated power $P_{\mathrm{rad}}(0) / P_{\mathrm{oh}}(0)$, on the inverse average drift speed. $P_{\text {oh }}(0)$ has been estimated to be $V_{s} / 2 \pi R \times j_{0}\left(q_{0}=0.95\right)$ where $V_{s}$ is the surface loop voltage. The time evolution is indicated by a solid line, and the shots terminating in a disruption are indicated by filled symbols. There is a greater spread in the axial data although there is no systematic dependence on the toroidal field or the plasma current. The spread may be due to the uncertainty introduced by the inversion procedure. The discharges which terminated in a disruption are now distinctly separated from the benignly terminated discharges by a "threshold" of $P_{\mathrm{rad}}(0) / P_{\mathrm{o} h}(0) \sim 15 \%$. This is below the limit of $80 \%$ within the $q(r)=2$ surface used by PERKins and Hulse (1985). The fact that the disruptions do not occur immediately on reaching a particular value of $P_{\mathrm{rad}}(0) / P_{\mathrm{o} h}(0)$ implies that any discharge which exceeds the value of $15 \%$ will eventually disrupt if the current and density are held fixed, although $15 \%$ can be exceeded for well over a confinement time. This appears as an "inevitability" criterion rather than a threshold. To illustrate that non-stationary discharges depart from the distribution found for stationary discharges, Fig. 6 shows two temporal evolutions as dotted lines. In the first case the reduction of the loop voltage to nearly zero led to an increase in $e N_{e} / I_{p}$, and the radiated power fraction was below the general distribution. In the second case, the gas feed was cut, resulting in a rapid drop in $e N_{e} / I_{p}$, and the radiated power fraction evolved above the distribution. It crossed the value of $P_{\mathrm{rad}}(0) / P_{\mathrm{oh}}(0)=15 \%$, and then dropped to more typical values during the benign discharge termination. Thus what appears to represent a disruption threshold. may be exceeded transiently. A discharge which evolves into a condition with a high probability of disruption, might be saved by a fast reduction in the plasma density.

This "apparent threshold" exists only for the "pure" deuterium discharges. Figure 7 shows a hatched band corresponding to the "pure" deuterium discharges of Figs. 3 and 6 and additional data from impurity puffing experiments. Quantities of $\mathrm{H}_{2}, \mathrm{He}$, $\mathrm{Ne}, \mathrm{N}_{2}$ and $\mathrm{O}_{2}$ were injected into a discharge, with $\bar{n}_{e}=2-3 \times 10^{19} \mathrm{~m}^{-3}$. The 

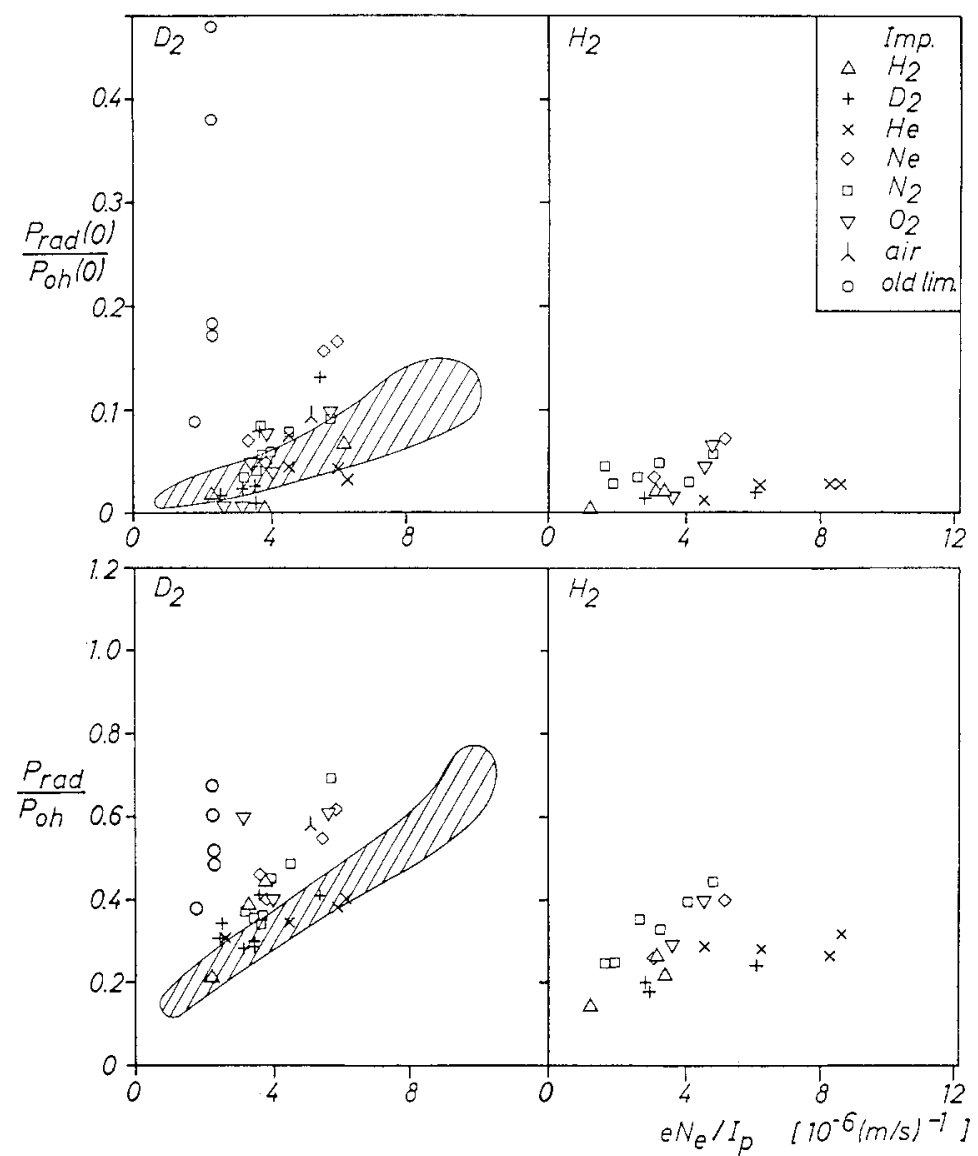

FIG. 7.-The normalised radiated power loss, total and axial, for impurity puffing discharges, and for discharges heavily contaminated by metallic impurities.

normalized total and axial radiated powers shown in the figure are different from the "generalized" form of the "pure" discharges. In addition Fig. 7 shows previous TCA data in which the metallic contamination of the plasma was high, and $P_{\mathrm{rad}^{-}}$ $(0) / P_{\text {oh }}(0)$ was $50 \%$ HofmanN et al., 1983). We conclude that the fairly narrowly defined distribution of the normalised power versus the reduced parameter $e N_{e} / I_{p}$, found for the "pure" discharges can not be generalised to other plasma conditions.

We have used the bolometric data to estimate the impurity concentration over a range of densities, for discharges at full current, with $T_{e} \sim 700-800 \mathrm{eV}$. We take the measured radial profiles of radiated power and electron density, and the modified coronal equilibrium radiation constants of Post et al. (1977). Since the low- $Z$ impurity radiation is small on axis for these values of electron temperature, we can calculate the metallic concentration using $\eta_{M}=P_{\text {rad }}(0) /\left(n_{e}(0)^{2} \times C_{M}\right)$ where $C_{M}$ is the radiated power loss per atom per electron. In the parameter range used, $C_{M} \sim$ $0.5 \times 10^{-25} \mathrm{Watt} /$ atom/electron. The density of the metallic impurities varies from $2-4 \times 10^{16} \mathrm{~m}^{-3}$ over the whole range of plasma density, with no obvious systematic dependence. This leads to a radiated power on axis which increases as $\bar{n}_{e}$. 
This increase in radiated power on axis has also been recently discussed by BAGDASOROV et al. (1985) and ISLER et al. (1985) following studies on the accumulation of injected test impurities in tokamak plasmas. Both studies concluded that there is enhanced impurity confinement towards the high density limit. BAGDASOROV et al. (1985) derived a confinement time increasing as $\bar{n}_{e} / I_{p}{ }^{2}$ whereas ISLER et al. (1985) found the increase in central impurity retention was as a function of $1 / B_{\varphi}$. Our results are in agreement with an increasing impurity confinement, although we cannot deduce this mechanism from the raw bolometric data.

\section{TEMPORAL EVOLUTION}

The value of $e N_{e} I_{p}$ fairly narrowly defines the radiated power loss for steady state discharges in one machine condition, i.e. filling gas, limiter type and cleanliness.

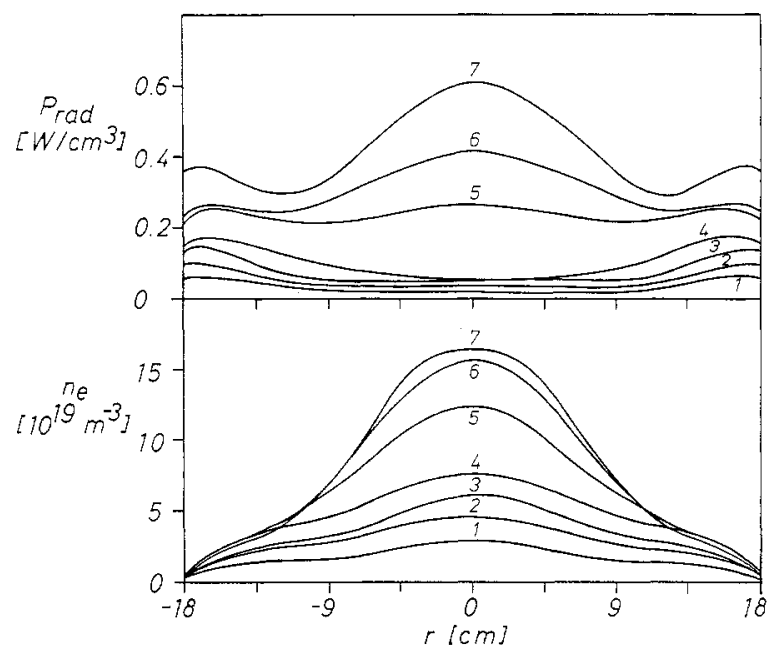

FIG. 8.-The temporal evolution of the local radiated power loss profile and the local electron density profile. ( $\mathrm{D}_{2}, B_{\varphi}=1.16 \mathrm{~T}, I_{p}=100 \mathrm{kA}$.)

Figure 8 shows the evolution of the inverted radial profiles of $P_{\mathrm{rad}}(r, t)$ and $n_{e}(r, t)$ through 7 points on the $e N_{e} / I_{p}$ scale, for the "pure" deuterium discharges, shown in Figs. 3 and 6 . The trend towards less hollow profiles as the inverse average drift speed increases is seen for all conditions. There is no evidence of a disruption precursor on the 16-channel bolometer, in terms of an abrupt discontinuous change in the radiated power profile. This only applies to the true high density limit, and not for discharges which terminate following intense Mirnov activity.

The multi-channel bolometer has been used to look at the radiated energy during a disruption. Figure 9 shows the radial profile of the time-integrated radiated power loss during a rapid current quench disruption, well within the density limit. We estimate the viewed radiated energy, i.e. the toroidally symmetric part, as $2.0 \mathrm{~kJ}$. The estimated stored kinetic energy of this discharge before the disruption was $1.3 \mathrm{~kJ}$ corresponding to $\beta_{p}=0.32$. A fraction of the stored magnetic energy, $11 \mathrm{~kJ}$, equal to the stored internal energy $L_{i} I_{p}{ }^{2} / 2$ plus the external magnetic energy $L_{0} I_{p}{ }^{2} / 2$, must be radiated during the disruption. Similar results were obtained by SCATURRo and PICKRELL (1979). 


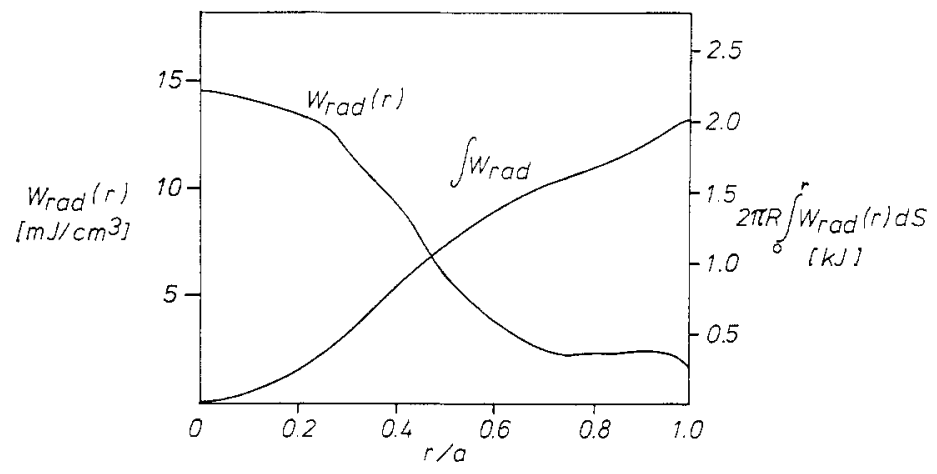

FIG. 9.-The time-integrated radiated energy loss during a disruption. $\left(\mathrm{D}_{2}, B_{\varphi}=1.51 \mathrm{~T}\right.$, $I_{p}=120 \mathrm{kA}, \bar{n}_{e}=4.7 \times 10^{19} \mathrm{~m} 3$.)

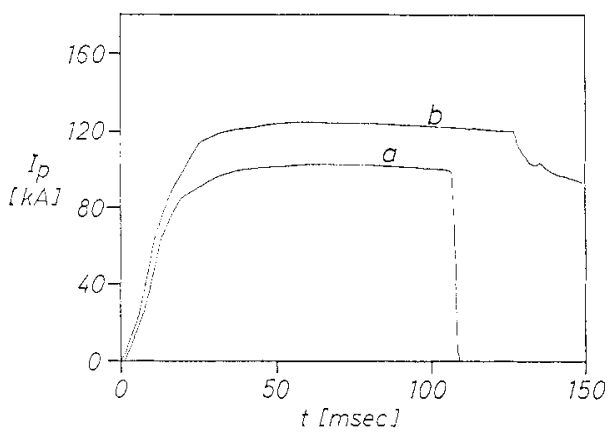

FIG. 10.--The time evolution of two different disruptions, (a) slow current quench and (b) a rapid current quench.

Figure 10 presents the time evolution of the plasma current for two discharges. Curve (a) represents the behaviour of a disruption often associated with $q=3$ or $q=2$, when the current is very rapidly quenched. This type of disruption can also occur well inside the operation diagram, and the data shown in Fig. 8 correspond to such a disruption, the current falling to zero within a few milliseconds. Curve (b) shows the typical behaviour of a high density limit disruption. The initial current quench is slow, and only $15-20 \%$ of the plasma current is lost before the position control system recovers. A considerable loss of both density and $\beta+1_{i / 2}$ occurs and the discharge then continues for $10-20 \mathrm{~ms}$ before control is usually again lost in a slow current quench $(20 \mathrm{~ms})$. We conclude that on TCA, the disruptions associated with the density limit are less severe than those at $q=2$ or $q=3$.

\section{CONCLUSION}

We have presented a wide range of data from ohmically heated discharges. For one set of conditions, the normalised total radiated power loss is a narrowly distributed function of the inverse average drift speed. When impurities are added to the discharge, this dependence is considerably altered. The occurrence of disruptions for given conditions correlates well with a threshold of the normalized axial radiated power loss. This value can be exceeded in impure discharges. No evidence of any 
disruption precursor has been seen on the multi-channel bolometer or on the multichannel interferometer. The metallic impurity density is a roughly constant function of the inverse drift speed. During a rapid current quench disruption a fraction of the stored magnetic energy is radiated away. In this study we have not found a simple relation between the radiated power and the occurrence of a disruption, which is valid for all conditions. We therefore conclude that it is highly unlikely that a simple model, based on an a priori threshold for disruptions, can explain our data.

Acknowledgements - We are grateful for the support and interest of Professor F. Troyon, and to B. P. DUVAL and F. B. MARcus for their comments on the paper. The work was partially supported by the Fonds National Suisse pour la Recherche Scientifique.

\section{REFERENCES}

Bagdasorov A. A., Bugarya V. I., Vasin N. L., Vershkov V. A., Vertiporokh A. N. et al, 12th European Conf. on Controlled Fusion and Plasma Physics, Budapest, Vol 9F, I, 207.

Gibson A. (1976) Nucl. Fusion 16, 546.

Hofmanin F., Hollenstein Ch., Joye B., Lietit A., Lister J. B., Nowak S., Peacock N. J., Pochelon A. and STAMP M. F. (1983) Lausanne report LRP 229/83.

JoYe B., Marmillod PH. and Nowak S. (1985) Lausanne Report LR P 285/86.

Isler R. C., Rowan W. L. and Hodge W. L. (1985) Phys. Rev. Lett. 55, 2413.

Lipschultz B., La Bombard B., Marmar E. S., Pickrell M. M., Terry J., Watterson R. L. and Wolfe S. M. (1984) Nucl. Fusion 24, 977.

Murakami M., Callen J. D. and Berry L. A. (1976) Nucl. Fusion 16, 347.

Niedermeyer H., Behringer K., Bernhardi K., Eberhagen A., Fussmann G. et al. (1983) 11th European Conf. on Controlled Fusion and Plasma Physics, Aachen 7D, I, 43.

Perkins F. W, and Hulse R. A. (1985) Physics Fluids 28, 1837.

Post D. E., Jensen R. V., Tarter C. B., Grasberger W. H. and Lokke W. A. (1977) Atomic Data and Nuclear Data Tables 20, 397.

Rebut P. H. and Green B. J. (1977), Plasma Physics and Contr. Nucl. Fusion Research (Proc. Int. Conf. Berchtesgaden 1976) 2, IAEA, 3.

RoBERTS D. E. (1983) Nucl. Fusion 23, 311.

SCAturro L. S. and Pickrell M. M. (1979) MIT-PFC/RR-79-8.

TLRNer M. F. and Wesson J. A. (1982) Nucl. Fusion 22, 1069.

Wesson J., Gowers C., Han W., Mast F., Nave F., Turner M. and Watkins M. (1985) 12th European Conf. on Contr. Fusion and Plasma Physics, Budapest, Vol. 9F, I, 147. 